\title{
TECCIENCIA
}

\section{Monitoring, Management and Topological Display Device using wireless network managed clients}

\author{
Monitoreo, gestión y visualización topológica de dispositivos gestionables de red mediante \\ clientes inalámbricos
}

\author{
Oscar Javier Melo Torres, ${ }^{1}$ Julio Barón Velandia. ${ }^{2}$ Alejandro Paolo Daza Corredor ${ }^{3}$ \\ ${ }^{1}$ Universidad Distrital Francisco José de Caldas, Bogotá, Colombia, ojato19@gmail.com \\ ${ }^{2}$ Universidad Distrital Francisco José de Caldas, Bogotá, Colombia, jbaron@udistrital.edu.co \\ ${ }^{3}$ Universidad Distrital Francisco José de Caldas, Bogotá, Colombia, apdaza@gmail.com
}

\begin{abstract}
The technological breakthroughs and developments made both online and on mobile devices has enabled the deployment of services that can be used by users without being in a fixed physical location or require transporting equipment with high computational processing power channel, this release has allowed people greater mobility and generating new business models based on e-commerce, to ensure an adequate service level a constant monitoring action is required to detect failures and shortcomings in timely manner without implying that staff is permanently in the management center . This paper presents the methodology, development and benefits of a prototype that allows visual access and ubiquitous real-time behavioral information and status of network devices from a mobile device requiring only a Web browser that can run the HTML5 and JavaScript protocol.
\end{abstract}

KeyWords: CSS3, HTML5, JavaScript, monitoring, network, topology.

\section{Resumen}

Los avances y desarrollos tecnológicos logrados tanto en Internet como en dispositivos móviles ha permitido la implementación de servicios que pueden ser utilizados por los usuarios sin necesidad de estar en una ubicación física fija o requerir el transporte de equipos con una alta capacidad de procesamiento computacional, esta liberación ha permitido a las personas mayor movilidad y la generación de nuevos modelos de negocio basados en el comercio electrónico, para garantizar un adecuado nivel de servicio es necesario realizar una constante acción de monitoreo que permita detectar las fallas y deficiencias de manera oportuna sin que esto signifique que el personal este permanentemente en el centro de gestión. En este documento se presenta la metodología, desarrollo y ventajas de un prototipo que permite acceder visualmente y en tiempo real en forma ubicua a la información de comportamiento y estado de los dispositivos de red desde un dispositivo móvil requiriendo únicamente que el navegador Web pueda ejecutar el protocolo HTML5 y JavaScript.

Palabras Claves: CSS3, HTML5, JavaScript, monitoreo, topología, red.

\section{Introduction}

Network management is understood as the set of processes and functions required for the management, maintenance, service provisioning in multi-vendor environment networking and communications systems [1]. The user in

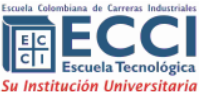

charge are divided depending on their functions, network managers are responsible for making constant monitoring, performing preventive maintenance and corrective as well as the generation and transmission of statistics analysis and network behavior. Meanwhile network administrators are responsible for managing the physical information of network devices, installed software, computer security, How to cite: Melo-Torres, O.; Barón- Velandia, J.; Daza- Corredor, A. , Monitoring, management and topological display device using wireless Network managed clients, TECCIENCIA, Vol. 7 No. 14., 93-100, 2013, DOI: http:/dx.doi.org/10.18180/tecciencia.2013.15.10 
distribution, interconnection and inventory. ${ }^{1}$

In a general environment, network administrators have a variety of tools that help them to keep detailed records of the elements of the network that are in charge of them. These tools make the focus groups according to which are developed and allow basically maintaining inventory of network devices, hardware and software information of computer equipment's, operating control devices remotely and management of users who access the network.

To facilitate this task, the administrators should then have a portable access user tool allowing detailed visualization of the most important network elements graphically placing them in a geographical environment which offers a reduction in the response time when carrying out corrective maintenance activity and which also allows monitoring the status of transmission of devices anytime and anywhere, providing performing other actions while the system remains on alert.

Most developments for network monitoring and management, provide a set of services that are configured to be accessed from a communication device or mobile phone using SMS text messages to provide such information when it presents disadvantages of operating in a network, which requires an additional contract with the operator of mobile communication. This monitoring functionality may be offered through parallel services or daemons ${ }^{2}$ that constantly inform customers previously registered in the mobile network status.

\section{System for monitoring and network}

The basic processes of maintaining office equipment and network devices are classified in two according to the methodology proposed for managing networks TMN (Telecommunications Management Network) [2] :

- Corrective maintenance: which is done after a not expected error occurs, in this case, the administrator must identify the network failure by a series of pre- arranged steps and detect the type of item that is causing the failure.

- Preventive maintenance actions to be performed in order to avoid problems that may be present in the future. A preventive activity usually suggests periodic review of the structure of the network and connections, check response times, updating antivirus and security software as firewall, proxies, monitoring, etc.

\footnotetext{
${ }^{1}$ This classification is based on the opinion of respondents experienced network administrators.

${ }^{2}$ From its acronym in English Disk and Execution Monitor Daemon, refers to a process or application that runs in the imperceptible to the user level.
}

Network administrators agree on the importance of constant monitoring of network using protocols and using applications that provide statistical traffic information. These capacities are overcome using, for example, protocols of application layer as SNMP (Simple Network Management Protocol) [3] and tracking applications as PING connectivity.

Table 1 shows the main features of the most important tools for monitoring network, as selection criteria were taking, Free Software, licensed software and businesses worldwide focus in the issue of network. As seen in table 1, the tools meet the requirements that are required for network management; however none of them present's visualization capabilities on mobile devices, only NAGIOS facilitates monitoring functions via text messages on mobile devices, being the most important asset of the prototype described here in.

Table 1. Management tools, network monitoring and services for mobile devices.

\begin{tabular}{|c|c|c|}
\hline \multirow{4}{*}{ TOOLS } & FEATURE & $\begin{array}{c}\text { MOBILE } \\
\text { DEVICE } \\
\text { DISPLAY }\end{array}$ \\
\hline \multirow{4}{*}{ NAGIOS $^{3}$} & Monitoring of network services & NO \\
\cline { 2 - 3 } & $\begin{array}{c}\text { User notification of events and } \\
\text { groups }\end{array}$ & NO \\
\cline { 2 - 3 } & $\begin{array}{c}\text { Notification to phones by SMS } \\
\text { (Short Message Service) }\end{array}$ & SI \\
\cline { 2 - 3 } & Autentification Scheme & NO \\
\hline \multirow{3}{*}{$\begin{array}{c}\text { NETWORK } \\
\text { MANAGER }\end{array}$} & WAN Monitoring & NO \\
\cline { 2 - 3 } & Hardware resources Monitoring & NO \\
\cline { 2 - 3 } $\begin{array}{c}\text { CISCO } \\
\text { WORKS }\end{array}$ & Alerts & NO \\
\cline { 2 - 3 } & Moports and Graphics & NO \\
\cline { 2 - 3 } & Monitoring Traffic Management & NO \\
\hline
\end{tabular}

Based on these characteristics, they are summarized as functional requirements for the development of a mobile solution topology display network devices, services monitoring, visualization through mobile devices and storage network traffic for the generation of alarms and awareness for registered customers.

\section{Prototype development}

\subsection{Structural Model \\ 3.1.1. Database Design}

For the management of persistence the relational database model was used due to its extensive documentation,

${ }^{3}$ NAGIOS. http://www.nagios.org/
${ }^{4}$ Manage Engine.
http://manageengine.adventnet.com/products/opmanager/index.html

${ }^{5}$ Cisco. http://www.cisco.com/en/US/support/index.html 


\section{TECCIENCIA}

simplicity and efficiency [4]. Specifically the design of the prototype Database provides several solutions for the correct functionality and coherence.

The Entity Relationship design of databases solves several important features required by the prototype, these features are described below:

- Users: All users in the system are recorded in the USER table to store basic information for identification and access to the prototype.

- 2. Profiles: As described before the prototype contains of three types of user profiles; Super Administrator, Administrator and Network Manager. As these types of user actors were considered for the development of functional requirements, the table that stores these profiles is called ACTOR.

- Permissions. Profiles also have a set of permissions that are reflected in the database as the relationship between actors and prototype services to which they have access. Since these services are available in the Web pages, such relationship between actors and the services are rendered as the link JSP resource [5] that encode or generate such Web pages. This relationship is available in the MODULE table, and direction of resources is recorded as relative addresses to the place where the prototype is installed.

- Device synchronization. This service carry out constant updating of information manageable assets devices, this information is stored and updated in the DEVICE table to differentiate the devices that are in transmission and the ones that not.

- Network Topology. The network topology is reflected in the database through the relation of 3 tables; LOCATION - DEVICE -CONNECTION, such relationship is summarized in the following semantic rule: each device is connected to another from different Locations.

\subsubsection{Structural Design}

In the structure of the prototype were used four different design patterns for the implementation; [6] for the construction of prototype model types [7]. The main concern was the simplicity of implementation with the speed to put into operation. Part of the functionality is based on other existing developments, Open Source, is the case of the GraphViz tool used as interface to represent the model of network nodes to monitor.

\subsubsection{Command}

Allows encapsulate all user requests and delegate them to the relevant objects. This prototype pattern is implemented to execute the ping and Telnet function in the case of interaction with the network, see Figure 1.

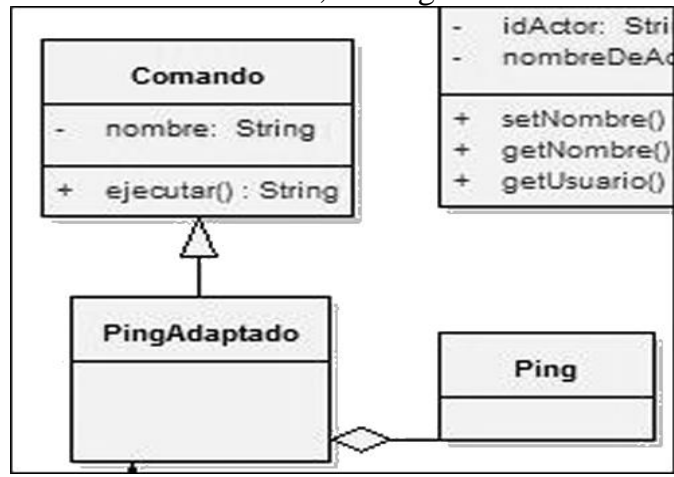

Figure 1. Implementing the Command design pattern.

\subsubsection{Facade}

Sometimes a library or set of libraries containing a set of functions to perform various actions repeatedly invoked by other libraries. In these cases, it is desirable to create an object that simplifies the API (Application Programming Interface) of the library, unifying and simplifying certain processes API. In this prototype pattern for centralized management restrictions are implemented, see figure 2.

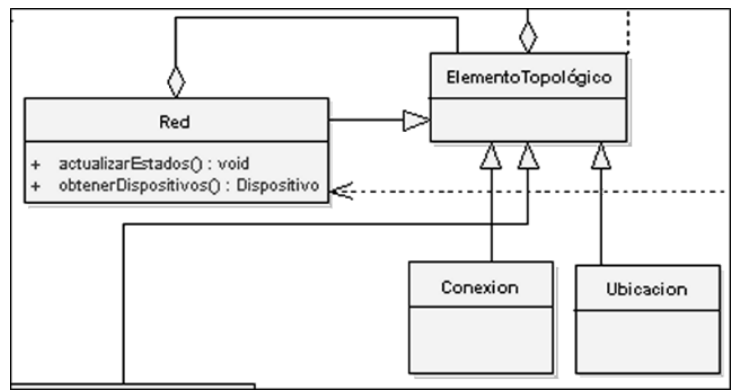

Figure 2. Implementing the Facade design pattern

\subsubsection{Composite}

Allows building a structure of elements that can be treated independent regardless of the state of these in terms of composition. It is essential the implementation of this pattern to form the network at runtime, allowing the topological states and display, see Figure 3. 


\section{TECCIENCIA}

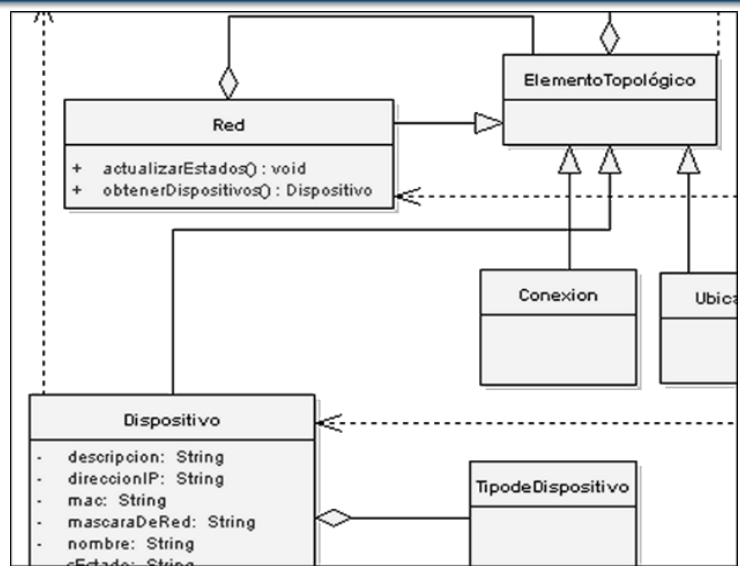

Figure 3 implementation of the Composite design pattern

\subsubsection{Adaptation DAO}

The DAO pattern is widely used for extensibility of persistent data, such as the independence of motor relational database (MySQL, ORACLE, SYBASE, etc.), for example. The use of the pattern facilitates to adapt easily to any system with the selected motor including only the kind that implements the code for each one of the enquiries, transactions, connection and disconnection. This pattern has several forms of implementation and each must create an object to perform select enquiries and transactions per persistent object, which would increase the complexity and the number of classes for the model. In the case of the prototype, was made a slight modification of this pattern to avoid creating numerous classes, centralizing all consultation processes and transactions into a single object (object persistence) and performing the translation to the selected motor database selected in the object type DAO that, for the case of the prototype is MySQL. See Figure 4.

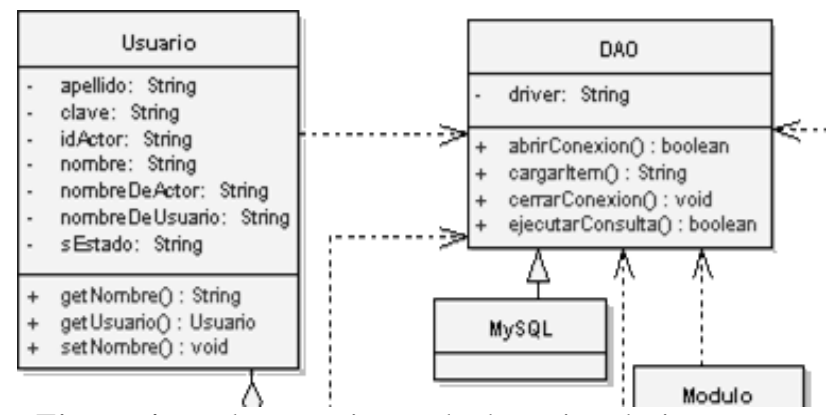

Figure 4. Implementation and adaptation design pattern DAO

\subsubsection{Dynamic Model}

The following describes the set of activities thar run the application and services through these generated. Activity diagrams [7] that follows are explained separately to understand the flow of activities and highlight the importance of each one.

\subsubsection{User Authentication}

The prototype performs user authentication for each activity or service that implements see Figure 5, in any case that authentication has run unsuccessfully returns the user to the access interface of the application.

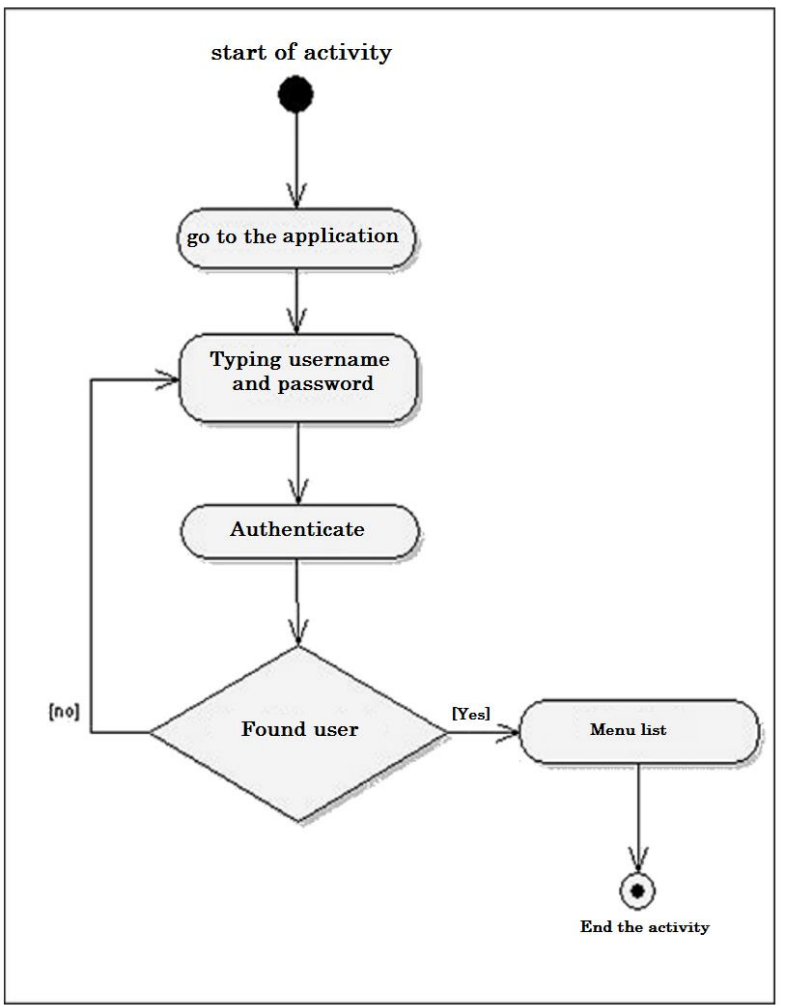

Figure 5. Activity Diagram "Authenticate User"

\subsubsection{User Management}

Like any information system prototype it has a module for managing users. The administration has given addition, modification and deletion, where each of these services is confirmed with messages.

\subsubsection{Viewing Devices}

The display device can be done using two methods: network topology and basic information available via SNMP, as identified in the prototype status display. It should be highlighted that the diagram of visualization topology contains active or sensitive areas representing each network node (device) allowing entry from there to the status display only selecting the device. See Figure 6. 


\section{TECCIENCIA}

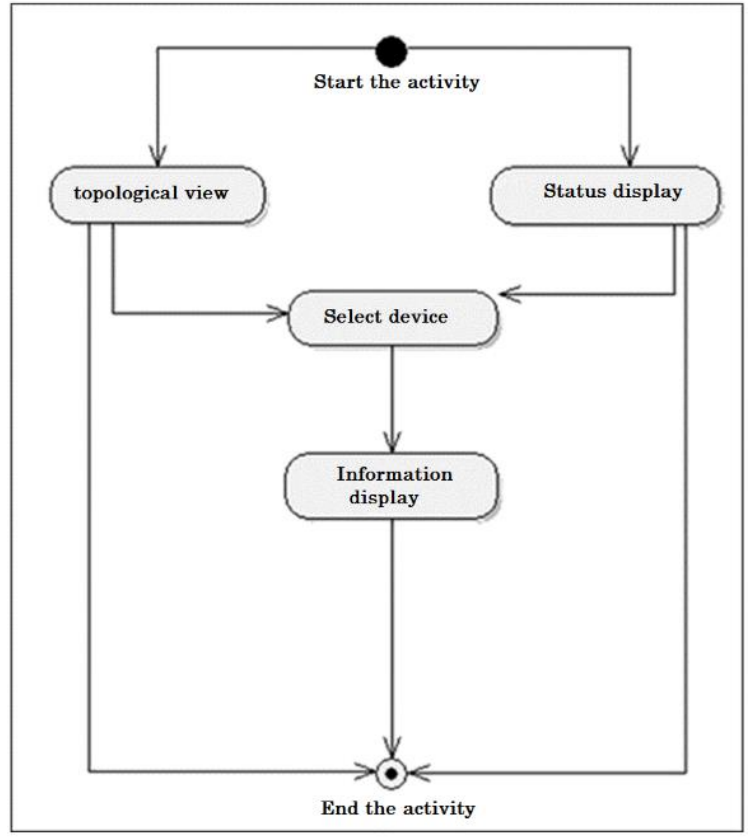

Figure 6. Activity Diagram "Display Devices"

\subsubsection{Monitoring devices}

This service display carries out 3 important activities that allows the administrator to easily monitor the behavior of the devices together or in a particular form. The first activity is the status display that can be run after entering the device or perform an automatic synchronization. The second activity is done alternating the flow of the application, is a daemon or permanent service that is configured in the operating system and of which the prototype uses to obtain constant information of statistics and dynamic updating of information by registered device through SNMP. Finally, the third activity can consistently generate synchronization with the devices; in this way to make a record or log of state per device in case of transmission states that are not expected. The latter is done as an alternative activity within the application, due to which requires user authentication but can also be programmed as a daemon or service operating system. See Figure 7.

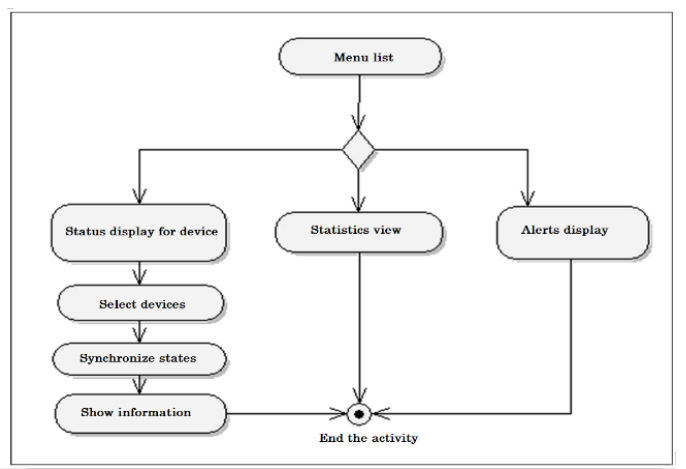

Figure 7. Activity diagram "Monitoring network devices

\subsubsection{Network Management}

Features the necessary functions for device management that allows to add, modify and delete functions of network devices, the accuracy of the data provides correct information of the topology display, status display, statistics and alerts. See Figure 8.

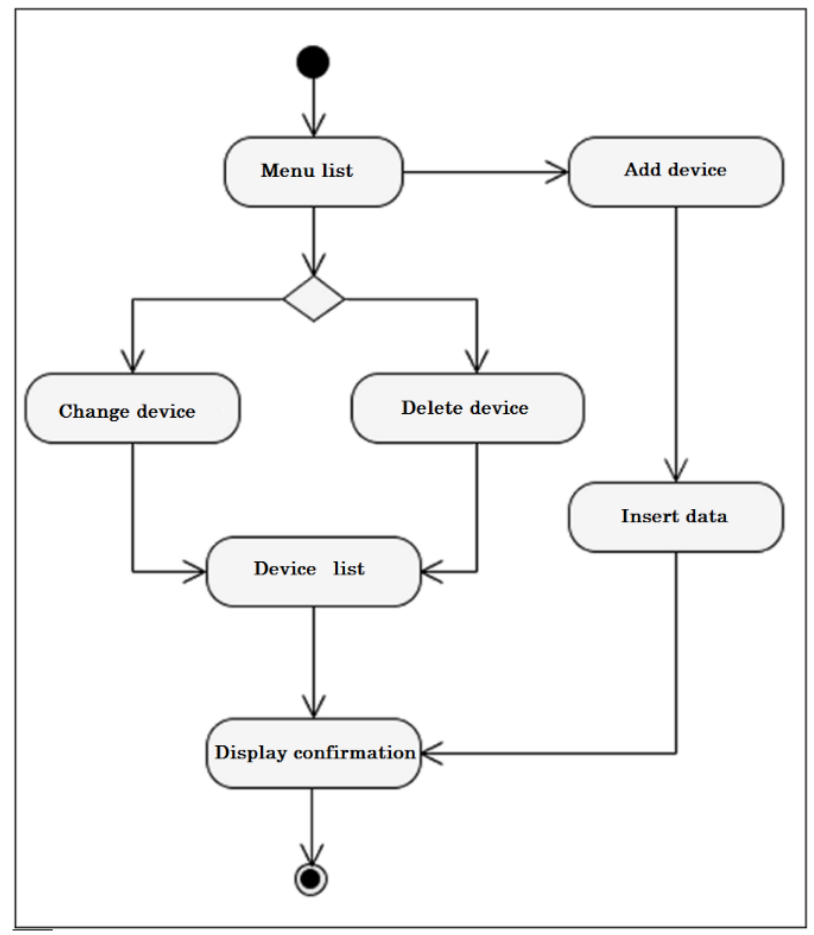

Figure 8. Activity diagram "Network Management"

\section{Integration and adaptation}

Some free software tools are widely used by network administrators, these tools must meet certain features to suit different environments, one of them and perhaps the most important is its extensive documentation in addition to the constant development of its versions.

\subsection{Network topology graph}

The prototype has a module to generate the topology of the network devices registered, this function is made possible by the structure of the database that stores the interconnection of devices network as already presented in section 3.1.1. The architecture of the prototype can adapt successfully to the Graphviz tool for the specific purpose of graph topology of the network, this is achieved through the Adapter design pattern. Once the prototype has generated the required file, this tool is invoked so that, depending on 


\section{TECCIENCIA}

the display ${ }^{6}$, a graph is generated in PNG or GIF format.

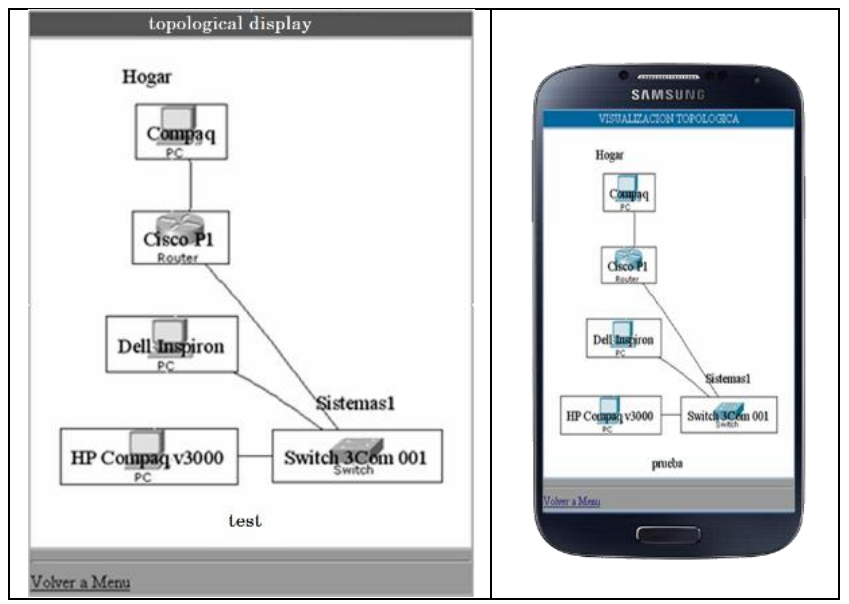

Figure 9. Network Topology: Graph with sensitive areas

Figure 9 shows the resulting output, to the left the generated graphics is seeing in a conventional WEB browser, while on the right is viewed from a mobile device using a navigator WEB device. The image is generated after calling Graphviz, is housed in a com-party images which are easily accessed by the prototype [8] file. Graphviz-ce also offers the advantage of mapping the network, that is to say that generates an HTML document [9], also based on the file generated by the prototype which distributes sensitive areas in the image, connections and devices. This feature allows viewing the image as the dynamic composition of all elements and not just as a static graphic, allowing selecting each network element for visualization, operation or modification to what is called network topology selection sensitive areas.

\subsection{Network Management}

For communication with manageable devices it is used the protocol SNMP allowing to obtain sensitive information management values such as network traffic and bandwidth.

To properly use, this protocol requires a manageable device, a management agent, and a network management system (NMS). For the prototype was used MRTG that has the function of extracting agent information in the device via SNMP where it should be using PERL for transparent execution of the tool. Figure 10 shows a graphic example of the resulting output of a monitored traffic for about 14 days where the gray light area represents the received data packets, while the black line represents the routed network.

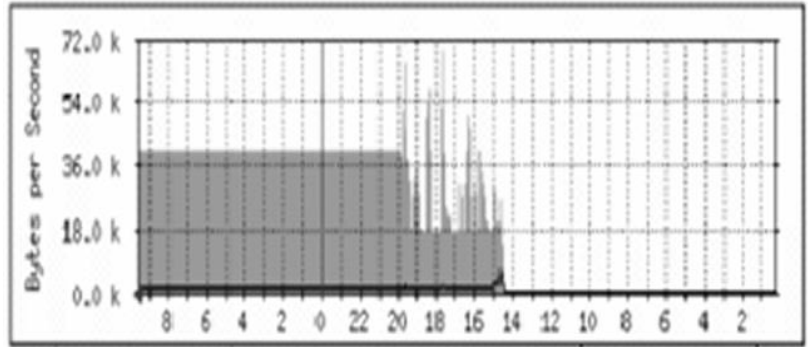

Figure 10. Graphical representation of network traffic generated by MRTG

For the integration of this tool with the prototype it is used the design pattern adapter that is responsible for sending information to MRTG of each manageable devices and maintains a background process running in PERL that is constantly aware of changes on this used information. The prototype maintains a trace of continuous synchronization between connected devices and the information obtained by MRTG, this information is captured and displayed to the user in addition the results are being stored in the database for future handling, as statistics network traffic.

Equally MRTG based on information provided by the prototype traffic generates images that are easily accessed and viewed by users. Quantitative information in this chart is summarized in a separate file and can be accessed easily allowing accurately present data to the user.

\subsection{Sensitive areas to display information}

The status display is achieved by implementing a fragment of JavaScript code used by Joomla ${ }^{7}$ for viewing sample images of their templates. This script was slightly adapted to the needs of the prototype for sensitive areas in the network topology and the status display. The script was implemented with the idea of showing a summary of information of the device on which points the mouse, showing the information that is considered relevant to the knowledge of the actual state of the device [10]. See Figure 11.

\footnotetext{
${ }^{6}$ From where you are accessing the user: WEB browser which for most mobile devices alone images in GIF are accepted.
}

\footnotetext{
${ }^{7}$ Management system dynamic portal engine and content management system, can be downloaded from: http://www.joomla.org.
} 


\section{TECCIENCIA}

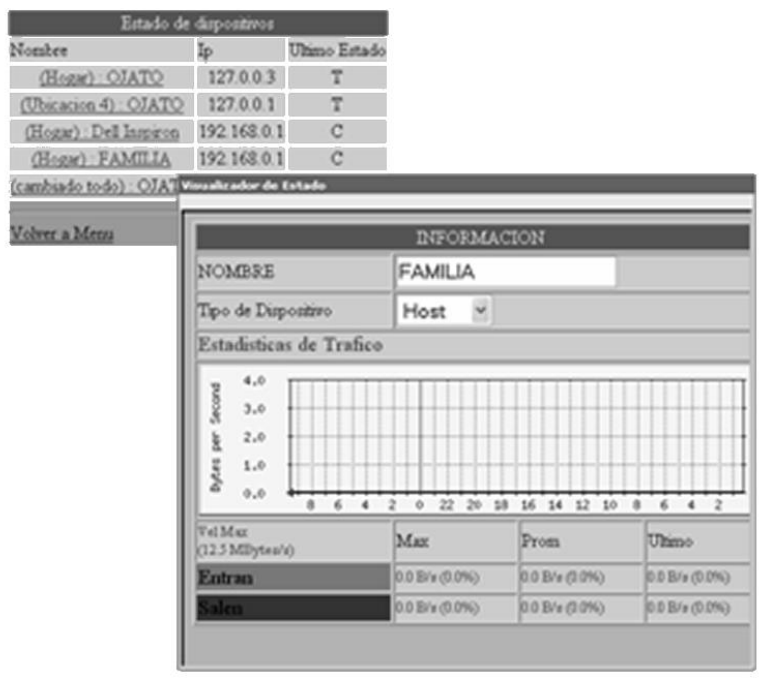

Figure 11. Temporary window that results to roll the mouse over the name of the device

\subsection{Ping and Monitoring States}

Depending on the environment in which is entering to the prototype, lifetime access implements a constant listening to the states as it's viewing. In the first case the prototype allows configuration time of monitoring in the file which is declared default to 60 seconds.

In a monitoring environment, this must maintain a continuous review of statements based on the defined time; this is achieved by updating the cache of the page during this time interval. This is a simple method, which in a Web environment; replace the need for implementation of parallel processes decreasing the complexity and development time.

Once the page has been updated, again invokes the function of monitoring the prototype which is an adaptation to the tracking application connectivity PING [11]. The adaptation is performed in such way that the prototype recovers all the output of this command, and depending on their response values and loss and also based on levels established control decides if the device is transmitting or if instead the connection has been interrupted.

Each of the faults that are present in the time of monitoring devices are registered in the database as alerts and can be visualized by transmission network at any time and from anywhere by logging even from their mobile devices that has the Internet browser . These alerts allow to be scheduled and controlled to perform a corrective control by the Network Manager and be registered as part of their work through the prototype.

\section{5 mobile content visualizacion}

To address the ability to present the graphic content of the prototype on a mobile device, the prototype has a unique Graphical User Interface, which is developed by using technologies such as HTML5, CSS3 and JavaScript that allow to use the model CSS3 Flexible Box adapting visualization to the different resolutions of mobile devices (W3C CSS3 Flexible Box Properties, 2012) (W3C, World Wide web Consortium. CSS Flexible Box Layout Module, 2013). The box model is based on a system of flexible containers that affect all HTML elements [9] with positioning not absolute of the containers obtaining the required adaptability

\section{Conclusions}

Navigation by mobile phones and devices will continue to grow in functionality and efficiency whenever the logic of the process is consistent with the capability of interaction and the availability of resources provided by these devices, when the applications are separate in layers the processing requirements are reduced on mobile devices, limiting their responsibility for the capture of interaction and graphical representation in the results of processing, this one is performed on the server, however depending on the capabilities of the browser implementation of the pattern Model View Controller; it would need the implementation of multiple visits, thus both time and cost will increase as well as the development, the product release, and the subsequent maintenance .

The current market provides a number of tools that together permit network managers administer efficiently the network based on the TMN approach, although it is a priority to use mobile resources such as devices and mobile phones that allow remote access and convenient services.

Software development by integrating components, allows the reuse of software modules while reducing the time required building new products. In the development of this prototype was essential to have different components provided by communities of free software, reducing development costs that facilitated the construction of a tool that integrates features for mobile network monitoring.

Object oriented programming to objects allows the translation of content to different environments and graphic representations, but this increases the number of classes and complexity because as it grows it must generate new objects that fit the content to these new environments, although adaptation is transparent to the developer who would only worry just to select the appropriate object.

The mobile access to the Internet enhances the possibilities of remote monitoring and control of various types of services that people want to implement, but its efficiency depends heavily on graphics capabilities and the possibility 


\section{TECCIENCIA}

of adequate translation of content, therefore it is important to consider the advantages provided by the flexible box model by integrating of CSS3 through the integration with HTML5 and JavaScript .

\section{References}

[1] A. Barba Marti, Gestión de red., Catalunya: Ediciones Universitat Politecnica de catalunya., 1999.

[2] A. Leinwand y K. Fang, Network Management: A practical perspective., Addison Wesley, 1993.

[3] S. J. Harnedy, Total SNMP: Exploring the Simple Network Management Protocol, Prentice Hall., 1998.

[4] J. Burch y G. Grudnitski, Diseño de sistemas de información:Teoría y Práctica, Mexico: Limusa, 1992.

[5] A. F. Quintas, Java server Pages: Manual De Usuario Y Tutoria,
Madrid: Ra-Ma, 2001.

[6] E. Gamma, R. Helm, R. Johnson y J. Vlissides, Design Patterns: Elements of Reusable Object-Oriented Software, Sydney: Pearson Education, 1994.

[7] C. Larman, UML y patrones: introducción al análisis y diseño orientado a objetos, Prentice Hall, 1999.

[8] A. Weitzenfeld, Ingeniería de Software orientada a objetos con UML, Java e Internet., Mexico: Thomson, 2005.

[9] T. A. Powell, HTML \& XHTML: The Complete Reference, Mc Graw-Hill., 2003.

[10] V. B, «MailXMail Técnicas para el desarrollo de computación móvil,» 1012 2012. [En línea].

[11] D. Comer, Interconectividad de redes con TCP/IP. Volumen II, Diseño e Implementación., Pearson, 2002. 